\title{
Chiral molecules split light: Reflection and refraction in a chiral liquid
}

\author{
Ambarish Ghosh and Peer Fischer \\ The Rowland Institute at Harvard, Harvard University, Cambridge, Massachusetts 02142
}

\begin{abstract}
A light beam changes direction as it enters a liquid at an angle from another medium, such as air. Should the liquid contain molecules that lack mirror symmetry, then it has been predicted by Fresnel that the light beam will not only change direction, but will actually split into two separate beams with a small difference in the respective angles of refraction. Here we report the observation of this phenomenon. We also demonstrate that the angle of reflection does not equal the angle of incidence in a chiral medium. Unlike conventional optical rotation, which depends on the pathlength through the sample, the reported reflection and refraction phenomena arise within a few wavelengths at the interface and thereby suggest a new approach to polarimetry that can be used in microfluidic volumes.
\end{abstract}

PACS numbers: 33.55.Ad, 42.25.Gy, 42.25.Lc, 78.20.Ek, 78.20.Fm, 78.30.Cp

Liquids composed of randomly oriented molecules are, in the absence of an external influence, isotropic, and are generally described by a single scalar refractive index. Optically active liquids, that is, chiral liquids which have the ability to rotate the polarization vector of light, are an important exception. These are characterized by two refractive indices, one for left- $(-)$ and one for right- $(+)$ circularly polarized radiation. Linearly polarized light may be regarded as a coherent superposition of left- and right-circularly polarized waves of equal amplitude, and Fresnel's theory of optical rotation shows that a difference in the respective indices of refraction causes the waves to acquire different phases as they propagate through the liquid and the polarization vector to rotate [1, 2]. The significance of optical rotation lies in the fact that it is a means to distinguish the two mirror image forms (enantiomers) of a chiral molecule. Most biologically important molecules are chiral and optical rotation is a well established analytical technique used to determine their absolute stereochemical configuration (handedness) in solution. Optical activity in liquids should, however, not only manifest itself through optical rotation in transmission, but should also be observable in reflection and refraction - as we demonstrate in this Letter.

If one considers the refraction of light at a boundary formed by a chiral and an achiral isotropic medium, as shown in Fig 1a, then the circularly polarized components must independently obey Snell's law:

$$
n_{0} \sin \theta_{0}=n^{( \pm)} \sin \theta^{( \pm)} .
$$

Circularly polarized light, incident with angle of incidence $\theta_{0}$ from the achiral medium characterized by the polarization independent refractive index $n_{0}$, will thus refract with angles of refraction $\theta^{(-)}$and $\theta^{(+)}$, depending on whether it is, respectively, left- or right-circularly polarized [1, 4, 5, 6]. Similarly, if unpolarized or linearly polarized light is incident from the achiral medium, then the light will split into two beams, one left- and the other right-circularly polarized [4, 7].

In order to verify that a linearly polarized light beam does split into its left- and right-circularly polarized components as shown in Fig [a, we imaged the separation be-
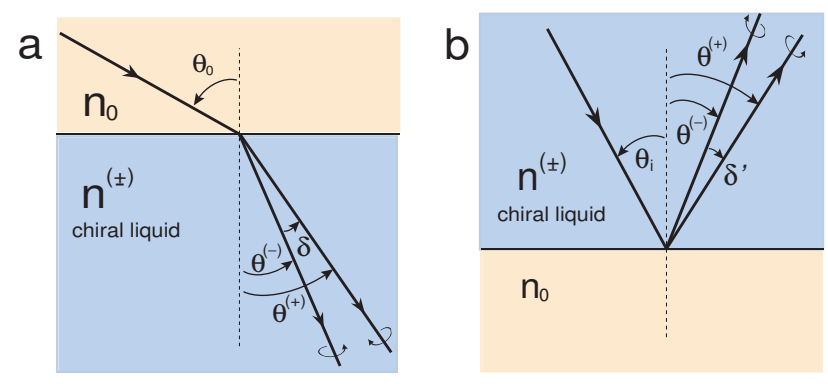

FIG. 1: (color online) (a) Refraction geometry at an achi$\mathrm{ral} /$ chiral interface. A wave incident from an isotropic achiral medium (angle of incidence $\theta_{0}$ ) is split into its circularly polarized wave components in the chiral medium. In an achiral medium the angle of reflection (not shown) does not depend on the polarization of the light and is therefore not sensitive to chirality. However, Silverman et al. showed that the specular reflection off a chiral surface can exhibit differences in intensity for the two circularly polarized components that depend on the circular dichroism (differential absorption) in the chiral medium [3]. Reflection geometry at a chiral/achiral interface in a chiral medium. A wave incident from a chiral medium is reflected at the interface and is split into its circularly polarized wave components. The transmitted ray (not shown) splits similar to the scheme shown in a).

tween the two refracted beams on a CCD camera. Given that the difference between the refractive indices is small, the angular divergence $\delta=\theta^{(+)}-\theta^{(-)}$between the refracted beams in Fig Ta can be written as

$$
\delta \approx \frac{\tan \theta}{n}\left(n^{(-)}-n^{(+)}\right)
$$

where $n=\left(n^{(-)}-n^{(+)}\right) / 2$ and where $\theta$ is the average of the two angles of refraction. The splitting needs to be large enough for it to be resolved on the detector. Fresnel showed that multiple-refraction at non-parallel interfaces can be used to increase the net divergence 1, [4]. Our experimental geometry, depicted in Fig 2], makes use of this amplification scheme, and is similar to the one originally devised by Fresnel to image double refraction 


\section{a}

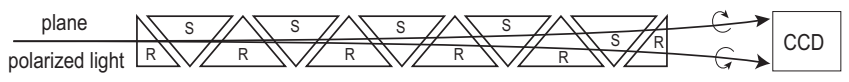

b

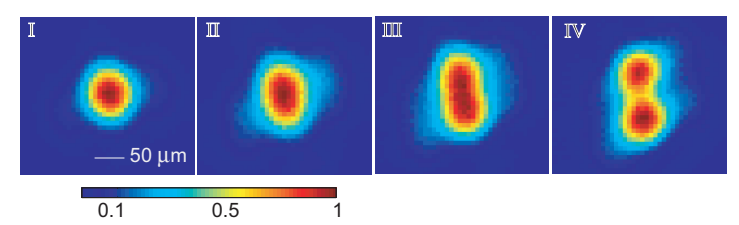

FIG. 2: (color online) (a) Schematic of the experimental geometry used to image double refraction. A plane polarized light ray is incident onto a stack of prismatic cuvettes, which are alternately filled with the R- and S- enantiomer of a chiral liquid. The angular divergence increases by $\sim 2 \delta$ at each $\mathrm{R} \backslash \mathrm{S}$ or $\mathrm{S} / \mathrm{R}$ interface, since both the order of the enantiomers and the orientation of the interface changes from one cuvette to the next. The beam would not split in an achiral liquid. (b) Intensity plots of a laser with Gaussian beam profile recorded on a CCD camera (4.6 micron pixel size). The images correspond to a passage through $8,12,16$, and 20 interfaces, (labeled I-IV respectively). Image IV clearly shows two well separated Gaussian beam profiles that are right- (upper) and left- (lower) circularly polarized.

in quartz crystals [1, 4, 5]. In Figure 20 it is seen that the splitting of a $405 \mathrm{~nm}$ diode laser beam, traversing prismatic cuvettes filled with optically active solutions of limonene, increases with the number of interfaces and becomes clearly resolvable on a CCD camera. We verified that the two beams in Fig 2b IV are indeed circularly polarized and that they are of opposite circularity. The refracted beams are of equal intensity and are coherent as the incident light is plane polarized. Should the incident light be unpolarized, then the two circularly polarized refracted beams have no fixed phase-relation [7].

Multiple interfaces amplify the double-refraction effects, since the angular deviation increases with each interface, but they are not required in order to measure the chirality-induced splitting of a light beam, which may also be observed in reflection. In the case of reflection inside a liquid, as shown in Fig पb, the angles can be obtained by considering the boundary conditions of the electromagnetic waves at the interface. The latter requires that the wavevectors of the incident $\vec{k}_{i}$ and the reflected beam $\vec{k}_{r}$ have the same tangential components along the interface, i.e. satisfy $k_{i} \sin \theta_{i}=k_{r} \sin \theta_{r}$, where the angle of reflection (measured from the interface normal) is $\theta_{r}$, and where $k_{r}=2 \pi n_{r} / \lambda$ and $n_{r}$ is the refractive index the reflected beam experiences. $k_{i}$ is correspondingly defined for the incident beam. An ordinary achiral liquid is described by a single refractive index and it follows that the law of reflection $\left(\theta_{r}=\theta_{i}\right)$ holds regardless of the polarization state of the light. Because a circularly polarized wave undergoes a (partial) polarization reversal upon reflection, the wavevectors for the incident and the reflected wave in a chiral liquid are associated with different refractive indices. Hence, in an optically active medium $\theta_{r} \neq \theta_{i}$, and there are two reflected waves with angles of reflection $\theta^{(-)}$and $\theta^{(+)}[8,[9,10]$. Their angular divergence after reflection is given by

$$
\delta^{\prime} \approx \frac{\tan \theta_{i}}{n}\left(n^{(-)}-n^{(+)}\right) .
$$

In general, the circular polarization components will not fully reverse their circularity upon reflection, but become elliptically polarized. The degree of circular polarization, and hence the fraction of the beam that doubly reflects, depends on the Fresnel reflection coefficients for a given interface, and needs to be accounted for in practice.

The divergence between the two circular components of a reflected or refracted beam can be directly compared with the optical rotation in radians developed by light at the wavelength $\lambda$ traversing a distance $d$ in a chiral liquid [2, 11]:

$$
\alpha=\frac{\pi d}{\lambda}\left(n^{(-)}-n^{(+)}\right),
$$

which is also a function of the circular birefringence $n^{(-)}-n^{(+)}$. It is thus expected that $\delta / \lambda$ and $\delta^{\prime} / \lambda$ will exhibit the same dependence on wavelength as optical rotation does, provided the dispersion of the average refractive index of the chiral medium in Eqs. (2) and (3) has been accounted for. To test this, we passed light from a Xe arc lamp through a monochromator and recorded $\delta$ and $\delta^{\prime}$ as a function of wavelength. The setup is schematically depicted in Fig 3. A photoelastic modulator is used to modulate the polarization of the light between left- and right-circularly polarized at $\sim 50 \mathrm{kHz}$ and the position of the light beam is synchronously recorded by a one-dimensional position sensitive detector and a lockin amplifier. Figure $3 \mathrm{~b}$ shows that the angular divergence measurements obtained in reflection as well as in refraction at a single interface between a chiral liquid and an achiral medium, are in complete agreement with the conventionally measured optical rotatory dispersion (ORD) spectrum. Our results show that reflection and refraction can be used in place of conventional optical rotation measurements, and their dispersion correspondingly in place of ORD for structural and stereochemical studies. The reflection data confirms that the 'law of reflection' does not necessarily hold in the presence of chiral molecules. Similar results have been obtained with other optically active liquids, such as pinene and aqueous glucose solutions.

Away from the interface, the bulk of a liquid does not affect reflection and refraction angles, and so it is interesting to ask if it is indeed possible to miniaturize the sample cell and hence the liquid volume without any loss of sensitivity. In doing so an important aspect is the shape of the liquid cell. The two circularly-polarized (cp) components will experience a small parallel displacement upon traversing a thin slab geometry with parallel front and exit windows, but will not experience any change in their propagation directions. The parallel displacement scales 
a

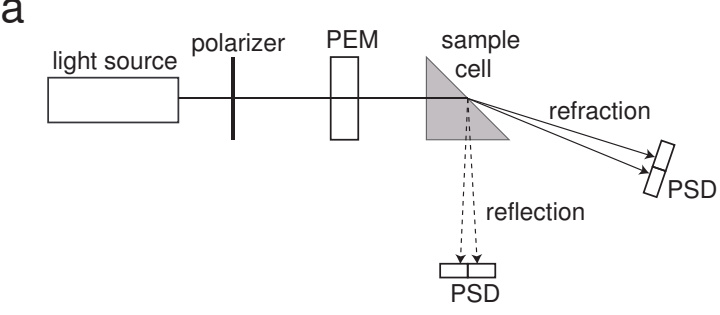

$\mathrm{b}$

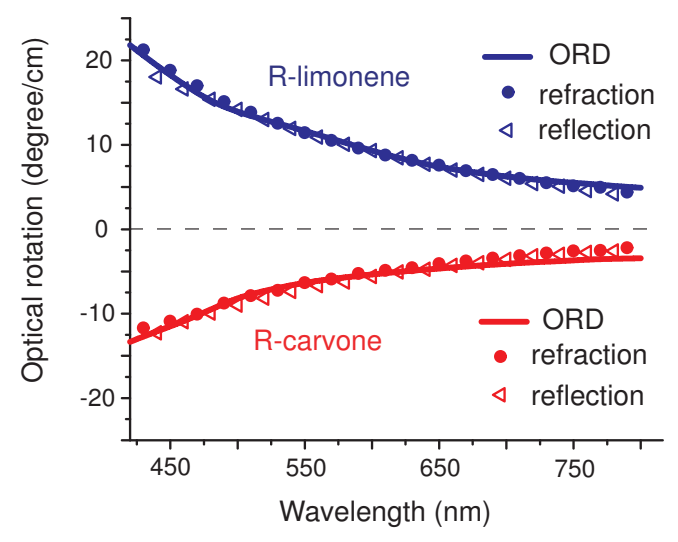

FIG. 3: (color online) (a) Schematic of the experimental geometry used for the reflection and refraction measurements, where PSD denotes a position sensitive diode and PEM a photoelastic modulator. In order to clearly separate reflection and refraction phenomena, a $90^{\circ}$ prismatic cuvette holds the chiral liquid, so that the doubly reflected beam exits the cuvette without doubly refracting. (b) Optical rotation and optical rotatory dispersion (ORD) data obtained from the wavelength-dependence of the measured angular divergence in both reflection (open triangles) and refraction (solid circles) at a single achiral/chiral interface for two optically active liquids $\mathrm{R}-(-)$-carvone (symbols in red) and R-(+)-limonene (symbols in blue). The data is seen to be in excellent agreement with conventional optical rotation measurements obtained with a spectropolarimeter (corresponding solid lines).

with the thickness of the fluid layer and this precludes miniaturization. The reflection/refraction must therefore take place in an asymmetric fashion, and in general, both phenomena will contribute to the angular divergence between the cp beams. We have constructed a micro-cell (inset Fig 4) where the liquid is confined asymmetrically by a part of a cylindrical Fresnel lens. In Figure 4 we compare the results from double refraction measurements in the micro-cell where the average path length through the chiral liquid is $\sim 400 \mu \mathrm{m}$ with results from a macroscopic cuvette where the light propagates $\sim 2 \mathrm{~cm}$ through an optically active liquid. A polarimeter would record an optical rotation in the micro-cell that is 50 times smaller than that of the macroscopic cell, yet in Fig \it is clearly seen that the measurements of the micro-cell and the macroscopic cuvette give rise to the same angular divergences. Since the circular birefringence of the chiral medium is proportional to the concentration difference between the two enantiomers, it follows that the angular divergence

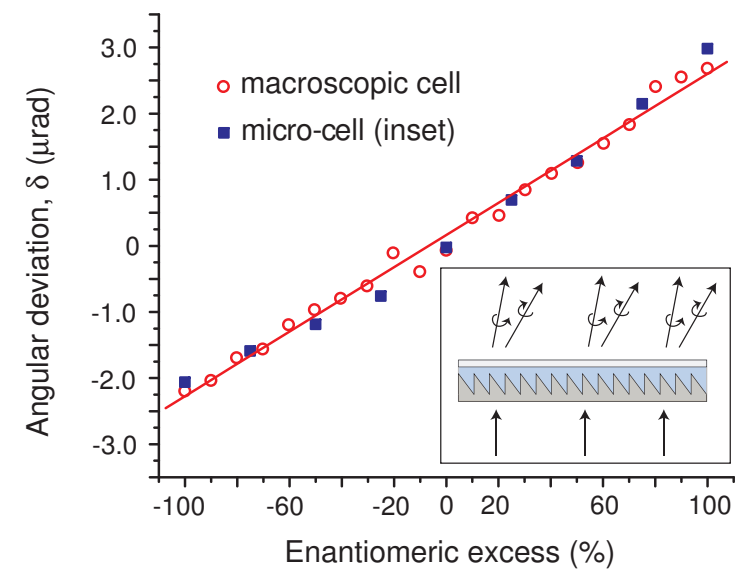

FIG. 4: (color online) Angular divergence $\delta$, measured in refraction, as a function of the concentration difference between the two enantiomers of limonene, denoted by $\mathrm{R}$ and $\mathrm{S}$, respectively. The enantiomeric excess is defined as the percent concentration difference between the enantiomers $\%[R]-\%[S]$. The measurements are obtained at a single achiral/chiral interface in two experimental geometries: a macroscopic cell (red circles) and a micro-cell (blue squares), with average path-lengths of $\sim 2 \mathrm{~cm}$ and $\sim 400 \mu \mathrm{m}$, respectively. Each data point is measured with a standard deviation of $\sim 10$ nanoradians and the straight line is a guide to the eye. The experimental setup used in conjunction with the micro-cell is shown in the inset: a thin film of liquid passes through part of a commercial Fresnel lens (height of $45^{\circ}$ sawtooth-prisms $\sim 700 \mu \mathrm{m}$ ), and a glass window separated by a $40 \mu \mathrm{m}$ spacer (not shown). The data sets have been obtained with a 488 $\mathrm{nm} \mathrm{Ar}+$ and a $405 \mathrm{~nm}$ diode laser and have been normalized to $488 \mathrm{~nm}$.

must be of equal magnitude and of opposite sign as the solution changes its handedness, and must be zero for the racemic (50:50) mixture, as is observed for both cells.

An attractive feature of the interfacial refraction and reflection phenomena is that the sample volume can be reduced further. If the optical pathlength in the microcell is reduced to a few microns, then already a modest [12] angular resolution of 100 nanoradians (well within the current absolute accuracy of the setup used in Fig (4) will make it possible to observe optical activities that are below the resolution limit of a standard laboratory polarimeter, i.e. $<0.001^{\circ}$. Miniaturization of optical components to micrometer dimensions is expected to give rise to diffraction phenomena. This immediately raises the interesting question whether the circular differential reflection and refraction phenomena of this Letter can also be observed in diffraction. Under normal incidence, the angular position of the diffracted spot at order $m$ for a diffraction grating with a groove spacing $D$ is given by $D \sin \beta=(m \lambda) / n$, where $n$ is the refractive index of the light in the medium where the diffraction grating is placed. Since the refractive indices of right and left circularly polarized light are different in an optically active medium $\left(n^{( \pm)}\right)$, the angular positions of the diffracted spots are also expected to differ. We have 
made measurements with reflection gratings and found that a chiral diffraction effect exists, such that left- and right-circularly polarized components diffract with different angles of diffraction [13]:

$$
D \sin \beta^{( \pm)}=(m \lambda) / n^{( \pm)}
$$

We believe that these findings together with our observation of double refraction and reflection in chiral liquids will open new possibilities in the detection of optically active molecules in small sample geometries such as in thin films or microfluidic devices.

The unique optical properties of chiral media have also been considered as components in electromagnetic coatings and microwave devices [14], and more recently, in connection with negative index media [15, 16]. Artificial structures such as chiral helices have been used to make optically active materials that give rise to relatively much larger optical rotations at microwave frequencies [17, 18, 19]. The double refraction and reflection phenomena that we have observed in liquids are therefore expected to give rise to correspondingly much larger angular divergences and splittings in chiral metamaterials.

Finally, it is interesting to note that a magnetic field renders any medium optically active, for a linearly polarized light wave propagating along the direction of the field will experience optical rotation. It thus follows, that the Faraday effect can also be observed in reflection and refraction [13] - similar to the chiral phenomena reported in this Letter.

The authors thank Drs. M. Burns, Y.-F. Chen, and J.M. Fournier for helpful comments, J. Park for the loan of a diode laser, and D. Rogers for the construction of a cuvette holder. Funding through the Rowland Junior Fellows program is gratefully acknowledged.
[1] A. Fresnel, Ann. Chim. Phys. 28, 147 (1825).

[2] L. D. Barron, Molecular light scattering and optical activity (Cambridge University Press, 2004), 2nd ed.

[3] M. P. Silverman, J. Badoz, and B. Briat, Opt. Lett. 17, 886 (1992).

[4] A. J. Fresnel, in Evres complètes d'Augustin Fresnel, edited by H. d. Sénarmont, E. Verdet, and L. Fresnel (Paris, 1822), vol. 1.

[5] T. M. Lowry, Optical rotatory power (Dover Publications, New York, 1964).

[6] M. P. Silverman, J. Opt. Soc. Am. A 3, 830 (1986).

[7] R. W. Ditchburn, Light (Dover Publications, New York, 1991).

[8] A. Lakhtakia, V. V. Varadan, and V. K. Varadan, J. Opt. Soc. Am. A 6, 23 (1989).

[9] M. P. Silverman and J. Badoz, J. Opt. Soc. Am. A 11, 1894 (1994).
[10] I. J. Lalov and E. M. Georgieva, J. Mod. Opt. 44, 265 (1997).

[11] E. U. Condon, Rev. Mod. Phys. 9, 432 (1937).

[12] We note that different detection schemes permit the resolution of 0.1 nanoradians, see for instance R.V. Jones, J. of Sci. Inst. 3837 (1961).

[13] A. Ghosh and P. Fischer, manuscript in preparation (2006).

[14] H. Cory, J. Electrom. Waves Appl. 9, 805 (1995).

[15] J. B. Pendry, Science 306, 1352 (2004).

[16] C. Monzon and D. W. Forester, Phys. Rev. Lett. 95, 123904 (2005).

[17] K. L. Lindmann, Ann. Phys. 63, 621 (1920).

[18] I. Tinoco and M. P. Freeman, J. Phys. Chem. 61, 1196 (1957).

[19] F. Guérin, PIER 9, 219 (1994). 